\title{
SILLAS FANTASMAS: UNA ANTOLOGÍA HEGEMÓNICA PHANTOM CHAIRS: AN HEGEMONIC ANTHOLOGY
}

Daniela Arias Laurino* Florencia Marciani Inés Moisset Zaída Muxí Martínez Un día una Arquitecta

\section{Resumen}

A través de la silla como mueble icónico que determinó momentos clave del diseño del siglo XX, el presente artículo analiza las antologías en términos de género con el objetivo de identificar el problema de la invisibilidad de las diseñadoras. Desvelar la ausencia de estas mujeres y hacer una revisión crítica sobre las historias de ellas, sus contribuciones y de las propias sillas, implica generar nuevos relatos que impidan la réplica de modelos y estereotipos en la profesión.

Palabras clave: diseñadoras, sillas, invisibilidad, exclusión, género.

\begin{abstract}
Through the chair as an iconic furniture that found key moments of 20 th century design, this paper analyzes the anthologies in terms of gender with the aim of identifying the invisibility of women designers. Unveil the absence of these women and make a critical review of their histories, their contributions, and the chairs they had design, involves a construction of new narratives in order to avoid reproducing stereotypes and professional roles.
\end{abstract}

Keywords: women designers, chairs, invisibility, exclusion, gender.

\footnotetext{
*E-mail: arias.03@gmail.com, arqmarciani@gmail.com, ines.moisset@gmail.com, zaidamuxim@gmail.com
} 


\section{Introducción}

Quizás ningún otro objeto de nuestro entorno cotidiano ha tenido un significado cultural tan duradero como la siempre presente silla, moldeando inconscientemente pero con fuerza las dimensiones físicas y sociales de nuestras vidas ${ }^{1}$.

Un estudio de compilaciones, antologías y colecciones de sillas del siglo XX, desvela la ausencia de diseñadoras. Cabe preguntarse si hay sillas producidas por diseñadoras y, si las hay, por qué no aparecen.

Desde el estado actual de la historiografía, el género se considera como una importante herramienta y categoría de análisis ${ }^{2}$. La construcción social sobre las diferencias sexuales ha sido institucionalizada en prácticas como las publicaciones tanto comerciales como académicas, prácticas que al día de hoy tienen impacto ${ }^{3}$.

"La epistemología feminista estudia lo anterior, abordando la manera en que el género influye en las concepciones del conocimiento, en la persona que conoce y en las prácticas de investigar, preguntar y justificar. Identifica las concepciones dominantes y las prácticas de atribución, adquisición y justificación del conocimiento que sistemáticamente ponen en desventaja a las mujeres porque se les excluye de la investigación, se les niega que tengan autoridad epistémica, se denigran los estilos y modos cognitivos femeninos de conocimiento, se producen teorías de las mujeres que las representan como inferiores o desviadas con respecto al modelo masculino, se producen teorías de fenómenos sociales que invisibilizan las actividades y los intereses de las mujeres o a las relaciones desiguales de poder genéricas, y se produce conocimiento científico y tecnológico que refuerza y reproduce jerarquías de género. La epistemología feminista hace ver estas faltas y propone diversas alternativas para resolverlas; explica por qué la entrada de mujeres feministas en las diferentes disciplinas académicas, especialmente en biología y en las ciencias sociales, ha generado nuevas preguntas, teorías y métodos; muestra cómo es que el género ha jugado un papel causal en estas transformaciones, y propone estos cambios como avances cognitivos y no sólo sociales, ya que la ciencia se ha distorsionado con supuestos sexistas en sus conceptos, teorías y aproximaciones metodológicas, por lo que es importante describir y considerar el contexto social, histórico, político y cultural en que se realiza la actividad científica" ${ }^{4}$.

El presente artículo se enfoca en la visibilización del trabajo de diseño de sillas de las diseñadoras pioneras y a lo largo del siglo XX que como en otras áreas de conocimiento sus aportaciones han sido eliminadas de la historia. 
En los últimos años se han incrementado los estudios que ponen en evidencia los sesgos de género en las profesiones relacionadas al diseño. Desde el equipo de Un Día | Una Arquitecta trabajamos desde 2015 para poner a disposición diaria en las redes sociales y hacer visible en idioma español, el aporte de las arquitectas en diferentes facetas ${ }^{5}$. De este modo, se trata de proponer una mirada crítica a la escritura de la historia desde puntos de vista exclusivamente masculinos. "Otra" mirada que cuestiona asimismo el impacto en cómo se ha desarrollado la práctica y el entendimiento científico de la objetividad partiendo de la base de que las comunidades científicas han estado integradas tradicional y principalmente por varones, de raza blanca y de clases privilegiadas. El trabajo abre las puertas para la construcción de una historia del diseño inclusiva donde las mujeres participaron activamente.

El siguiente artículo busca identificar sesgos de género en el armado de antologías vinculadas al diseño de mobiliario durante el siglo XX. Su propósito es diagnosticar el problema de la invisibilidad de las diseñadoras para poder intervenir y no reproducir los estereotipos vinculados a la profesión.

La investigadora y profesora de lengua y literatura Elaine Hobby que trabajó sobre antologías de escritores dice lo siguiente:

"Cuando empecé este proyecto en 1979 "simplemente" quería investigar sobre las mujeres escritoras olvidadas, porque estudiar literatura inglesa significaba casi únicamente estudiar la escritura de los hombres. Sólo quería saber qué había y quería compartir mis hallazgos con otras mujeres [...] A medida que avancé en el trabajo mi perspectiva cambió [...] Cuando empecé este estudio estaba trabajando sobre "mujeres olvidadas". Cuando terminé, me preocupaba el problema de lo que les sucede a los grupos subordinados que viven bajo regímenes reaccionarios" 6 .

Hemos elegido trabajar con las sillas por su carácter icónico y por la disponibilidad de material referido al tema que permite hacer un análisis pormenorizado. No se han encontrado trabajos específicos realizados con estos datos.

Se inicia trabajando con indicadores cuantitativos básicos que ponen en evidencia la presencia de diseñadoras en ámbitos de representación como publicaciones, exposiciones y catálogos de empresas especializadas relevantes. En principio se trata de contabilizar la cantidad de diseñadoras versus la cantidad de diseñadores en estos ámbitos para, posteriormente, poder además clarificar cómo han sido citadas las autorías, si son correctas, si mencionan a la totalidad del equipo. Los datos obtenidos permiten visibilizar y medir la magnitud del fenómeno de la desigualdad de género, y aproximarnos a sus causas. Si la visibilidad no se mide es un problema que no existe.

Por otro lado se realiza un mapeo, organizado como línea de tiempo donde se indican piezas de mobiliario que no están en las antologías pero que perfectamente podrían haber estado. 
Por último se hace una revisión crítica de las historias de estas sillas y de sus diseñadoras, de sus trayectos de formación, de los reconocimientos y de las dificultades que encontraron para ser reconocidas, (poniendo especial atención en sus experiencias contadas en primera persona) para llegar a algunas conclusiones provisionales sobre el tema.

\section{Publicaciones, Exposiciones y Catálogos}

Los distintos medios para la divulgación de los objetos de diseño y en particular de las sillas han sido de fundamental importancia para su desarrollo. Mediante la difusión de una selección de piezas determinadas se genera influencia sobre el colectivo profesional (artístico y empresarial) que influye, a su vez, con su demanda y sus creaciones. Esto genera una consolidación de valores, es decir, se orienta sobre qué es lo bueno, qué es lo bello, etc.

La observación de una sola pieza, la silla, nos permite hacer un recorte y desarrollar una metodología de trabajo que luego podría ser replicada. En primer lugar, seleccionamos dos publicaciones, la publicación de la exposición 100 sillas clásicas del Vitra Design Museum (1997) ${ }^{7}$ y el catálogo Chairs: Catalogue of the

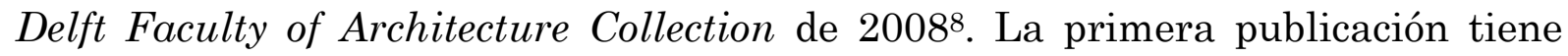
una clara orientación comercial ya que está destinada a la venta de sillas en miniatura ${ }^{9}$. La segunda, supone el inventario de una colección académica con fines pedagógicos, ya que releva el acervo de sillas en la Escuela de Arquitectura de Delft ${ }^{10}$.

Si revisamos con la metodología de indicadores cuantitativos diferenciados por sexo las publicaciones dedicadas al diseño de mobiliario y de sillas en particular encontramos que:

De las 100 sillas que presenta la publicación de Vitra, sólo dos mujeres aparecen en el índice: Charlotte Perriand y Ray Eames. Se menciona con voz propia el diseño de la silla Ombre (1953-1955) de Charlotte Perriand y de autoría compartida, cinco diseños ${ }^{11}$ de Charles y Ray Eames. Se agregan también a la autoría compartida entre Le Corbusier, Pierre Jeanneret y Charlotte Perriand (en ese orden) las sillas B301, B302 y B306. Como veremos más adelante, esta autoría compartida es un ejemplo de apropiación y marketing.

Hay claras omisiones de mujeres arquitectas y diseñadoras pero no de sus socios, como el caso de Lilly Reich, Aino Aalto, Denise Scott-Brown o Margaret MacDonald. Esta última sólo aparece mencionada en el texto que describe la silla Hill de manera secundaria.

En el catálogo de Delft, aparecen Maria Tenicheva, Ida Falkenberg y Ray Eames entre 59 autores. Aino Marsio vuelve a ser omitida de la autoría de cinco piezas presentadas como solamente de Alvar Aalto. Ray Eames aparece en cinco piezas en autoría compartida con Charles Eames y en una no ${ }^{12}$. De la misma manera que en la otra publicación, la presencia de diseñadoras autoras de sillas es extremadamente baja ${ }^{13}$. 


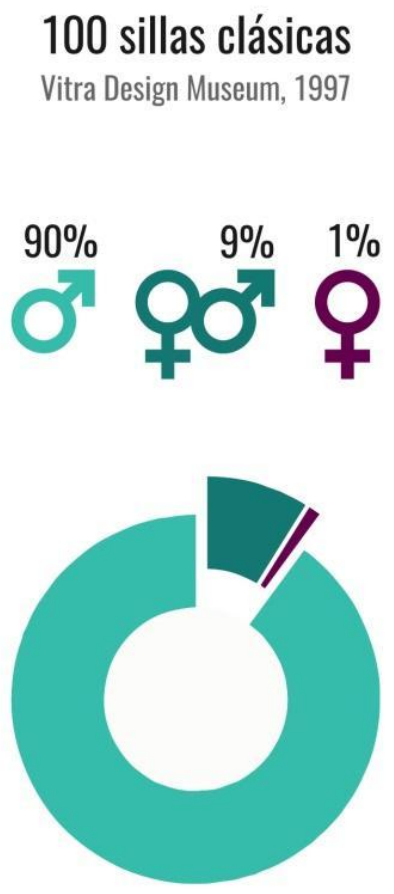

Chairs
Catalogue of the Delft Faculty of Architecture Collection
Máčl, Otakar; Woertman, Sander; Van Wijk, Charlotte
010 Publishers, 2008
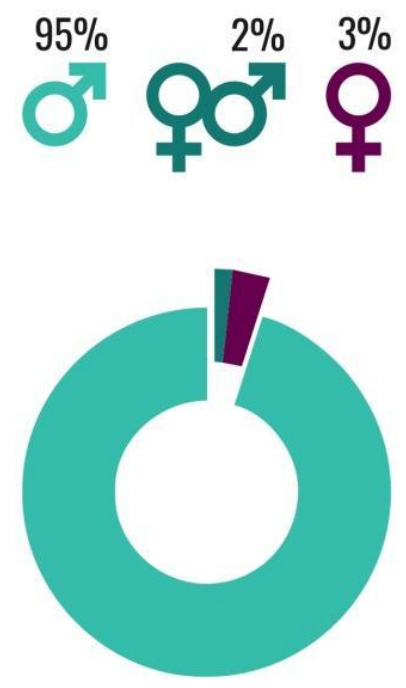

Fig. 1. Gendercheck de las publicaciones 100 sillas clásicas del Vitra Museum (1997) y el catálogo académico Catalogue of the Delft Faculty of Architecture Collection (2008). Elaboración de Florencia Marciani, enero 2017.

Estas cifras se reflejan en los libros académicos referidos al tema. Por ejemplo, en el libro An Introduction to Design and Culture, 1900 to the Present de Penny Sparke publicado en 1986 y reeditado en 2004 y 2013 se hace referencia principalmente a tres diseñadoras modernas de muebles; Eileen Gray, Charlotte Perriand y Lilly Reich ${ }^{14}$. Si bien la autora reconoce que hay una historia oculta que invisibiliza a las mujeres ${ }^{15}$, ésta publicación se centra en la evolución del diseño desde la modernidad, época en que la figura del diseñador profesional adquiere relevancia, y el discurso al igual que en The Modern Interior, gira en torno a la figura masculina. La mayoría de las diseñadoras citadas se dedican a las artes decorativas (moda, textiles, cerámica, etc.), mientras que ellos son los que realizan las innovaciones sobre todo las tecnológicas. Se omiten diseñadoras como Aino Marsio o Denise Scott Brown o se minimiza el trabajo de Ray Eames, Charlotte Perriand o Lilly Reich como se ha descrito. Por otra parte, el diseño es visto desde una perspectiva europea y estadounidense (eventualmente Japón) lo que refuerza la noción de falsa objetividad en la investigación, siempre existirá una mirada, una situación socio-política, un punto de vista personal o colectivo ${ }^{16}$.

¿Qué sucede en los espacios de la cultura? Desde el pasado mes de diciembre de 2016 y hasta el próximo mes de abril de 2017 el MoMA abre sus puertas a la exposición How Should We Live? Propositions for the Modern Interior. La exposición se centra en los espacios interiores de varios diseñadores del Movimiento Moderno y hace hincapié en proyectos de mobiliario mujeres arquitectas-diseñadoras como Eileen Gray, Charlotte Perriand, Lilly Reich o 
Grete Lihotzky. De similares características pero dedicada exclusivamente a las mujeres diseñadoras durante un siglo el MoMA presentó en 2013 y 2014 Designing Modern Women 1890-1990.

En 2013 el Centre Pompidou expone la obra completa de Eileen Gray, una retrospectiva inédita que recogió, con el mismo interés, su arquitectura, mobiliario, pinturas y fotografías ${ }^{17}$. De la misma forma, organizada por Matilda McQuaid, se expuso en el MoMA la contribución de Lilly Reich de manera exclusiva, Lilly Reich: Designer and Architect (1996).

Aunque exponer el trabajo de estas arquitectas constituye un avance en cuanto al reconocimiento que aportan, no dejan de ser muestras anecdóticas para lo que culturalmente suponen los museos como espacios de representación y difusión. Como señala la artista Haizea Barcenilla sobre la exposición de Eileen Gray:

“... el sistema de arte se basa en legitimaciones y por mucho que haya muy buenos argumentos que digan que Eileen Gray era una gran arquitecta, para la opinión popular la confirmación llega cuando un centro de referencia dedica sus salas a exponerla"18.

Estos últimos años en ocasión del cincuentenario de la muerte de Le Corbusier se realizaron varias exposiciones donde se incluyó el trabajo de Charlotte Perriand ${ }^{19}$ ni de otros colaboradores y colaboradoras y consolidando así la figura del héroe.

De manera soslayada aparecen las mujeres diseñadoras en exposiciones del MoMA como Bauhaus 1919-1933: Workshops for Modernity (2010) o Counter Space: Design and the Modern Kitchen (2010-2011) donde se exhibe la transformación de la cocina del siglo XX y se pone de relieve la cocina de Frankfurt de Grete Schütte-Lihotzky.

Las exposiciones dedicadas a las mujeres artistas en general, son abrumadoramente minoritarias en relación a las destinadas a los hombres pero lo son infinitamente menores aquellas reservadas a las mujeres de las vanguardias $\mathrm{y}$ a las arquitectas.

El escasísimo protagonismo de las mujeres en grandes museos y centros dedicados al arte y la cultura es consecuencia de un sistema (del arte y el diseño) construido en base a valores patriarcales que ha excluido a las mujeres de este sistema y por tanto de colecciones y catálogos.

Según Joan Wallach Scott ${ }^{20}$ no es la falta de información sobre las mujeres sino la idea de que tal información no tenía nada que ver con la historia lo que condujo a la invisibilidad de las mujeres en los relatos. En este sentido, como señala la Crítica y Profesora de Historia del Arte Haizea Barcenilla no es que los museos tengan menos obras de mujeres para exponer sino que no han accedido a ellas porque, entre otras cosas, el diseño o las artes decorativas se consolidaron históricamente como artes menores.

Desde el punto de vista de los medios de producción del diseño, si analizamos en la actualidad los catálogos de empresas de muebles como Cassina, 
Knoll o Artek, podemos ver la perpetuidad en los mecanismos de exclusión a pesar de algunos esfuerzos ${ }^{21}$.

La mítica compañía de diseño Cassina, fundada en 1927, se lanzó en los años 50 al diseño industrial en Italia pasando de la artesanía a la producción en serie. De un total de 49 sillas que recoge la colección en su catálogo actual ${ }^{22}$, 8 son sillas diseñadas por Charlotte Perriand ${ }^{23}$ y 2 de autoría compartida con Le Corbusier, Pierre Jeanneret. Esta empresa produce 3 modelos a los que adjudica la autoría exclusiva a Charles Rennie Mackintosh, incluyendo la silla Hill que igual que las publicaciones excluye el trabajo de Margaret MacDonald.

De la misma forma, los indicadores cuantitativos de diseñadores y diseñadoras en los catálogos de Knoll y Artek (Propiedad de Vitra en la actualidad) muestran una presencia minoritaria de sillas diseñadas por mujeres. Es notable que en la totalidad de la lista de sillas y sillones Artek, no aparece una sola mujer ${ }^{24}$. El catálogo de Knoll25, sin embargo, es, de los tres analizados, el que presenta mayor presencia femenina en los diseños que produce. Cabe la interrogante si esto se debe a la presencia durante mucho tiempo de Florence Knoll al frente de la empresa la que también incorpora diseñadoras y equipos jóvenes.

Cassina

Diseñadores
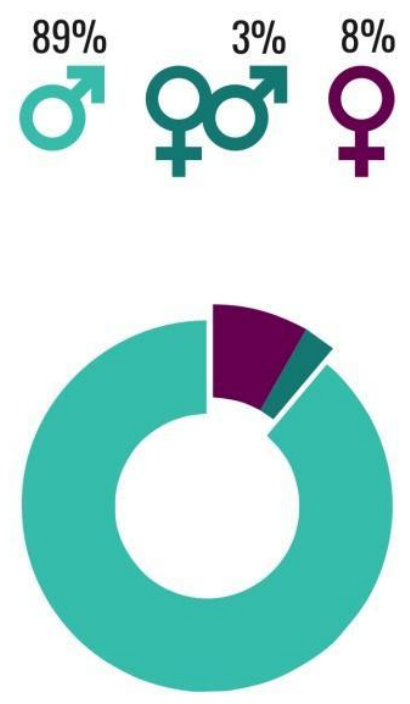

Knoll

Diseñadores
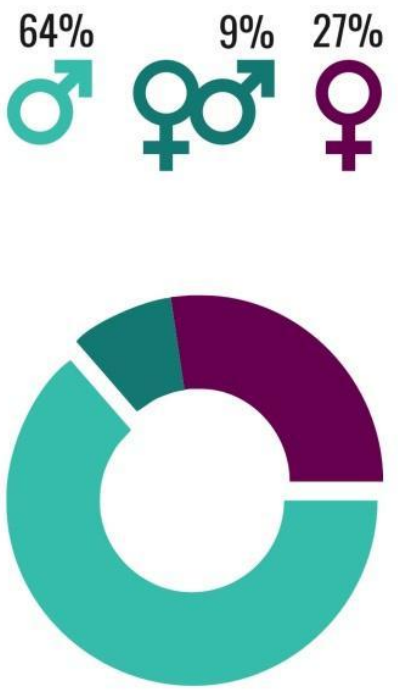

Artek

Diseñadores
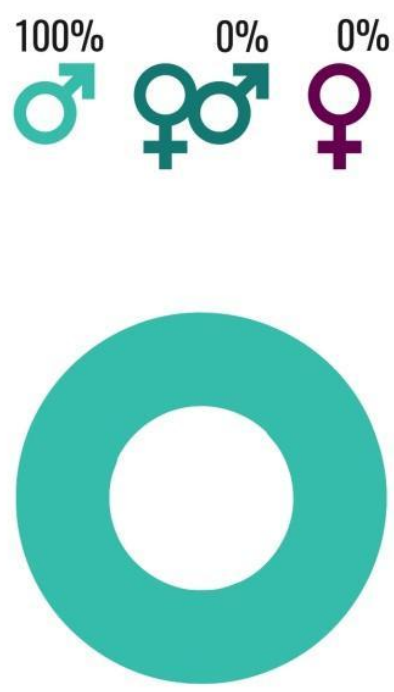

Fig. 2. Gendercheck de los catálogos de las empresas Cassina, Knoll y Artek. Elaboración de Florencia Marciani, enero 2017.

La publicidad forma parte de las prácticas de las empresas para promover sus diseños. Por eso es imprescindible entender las complejas interacciones entre los medios de producción y comunicación y cómo convergen además con los espacios académicos y de representación (exposiciones y premios). Durante el 
siglo XX los medios impulsaron prácticas sociales para instalar la modernidad en la vida cotidiana. La divulgación de lo cotidiano hizo referencia a dos variables; el rol del sujeto como ser social y el escenario donde se desarrolla como lugar de acción.

La difusión y publicidad de la silla como objeto funcional y de diseño es capaz de reflejar cada tiempo histórico: cambios sociales, tecnológicos y estéticos. Los nuevos materiales como el plástico, tubos de acero, etc., y los procesos de producción en serie fueron factores trascendentales en el desarrollo comercial de estos productos. Pero así como promovían las novedades desde el punto de vista tecnológico, los avisos y catálogos reproducían y sostenían también estereotipos sexistas. Las empresas no citaban a las diseñadoras como autoras.

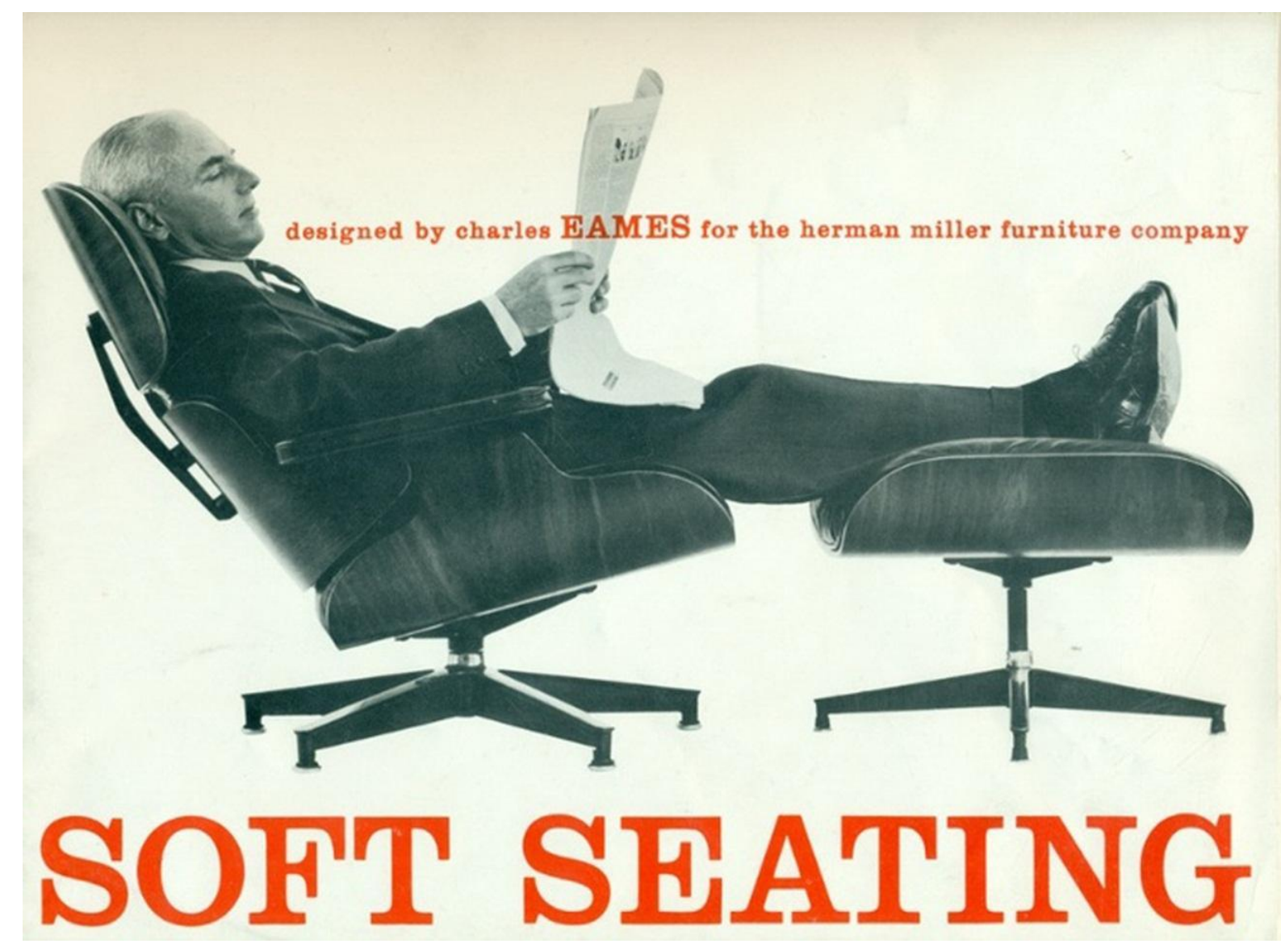

Fig. 3. "Design by Charles Eames". Publicidad de Lounge Chair \& Ottoman de la empresa Herman Miller omitiendo la autoría de Ray Eames. Campaña soft Seating, 1959.

Fuente: http://www.hermanmiller.com/products/seating/lounge-seating/eames-lounge-chair-and-ottoman.html

Por otro lado, las imágenes que presentan a las mujeres en relación a las sillas, ni es neutra, ni es inocente y fomenta estereotipos y roles de género así como modelos de comportamiento sexista. Así, encontramos mensajes con un 
discurso dominante, que margina a las mujeres al espacio doméstico ${ }^{26}$ (al matrimonio, al cuidado de la casa, el marido y los hijos) o en el otro extremo donde la publicidad del producto está vinculado al desnudo femenino ${ }^{27}$.
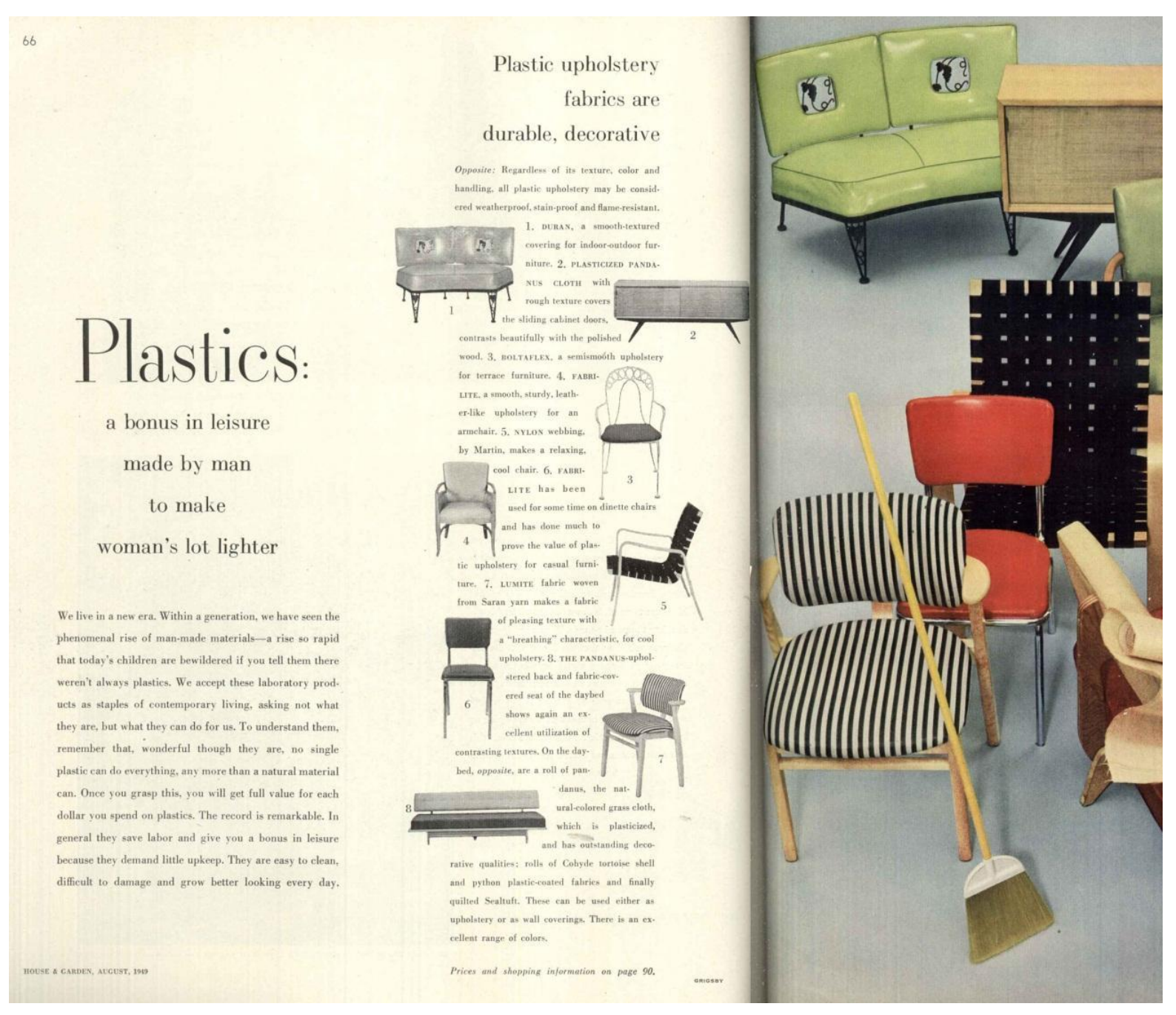

Fig. 4. Artículo "Creado por el varón para hacérselo a las mujeres mucho más liviano", revista House \& Garden, agosto, 1949. 


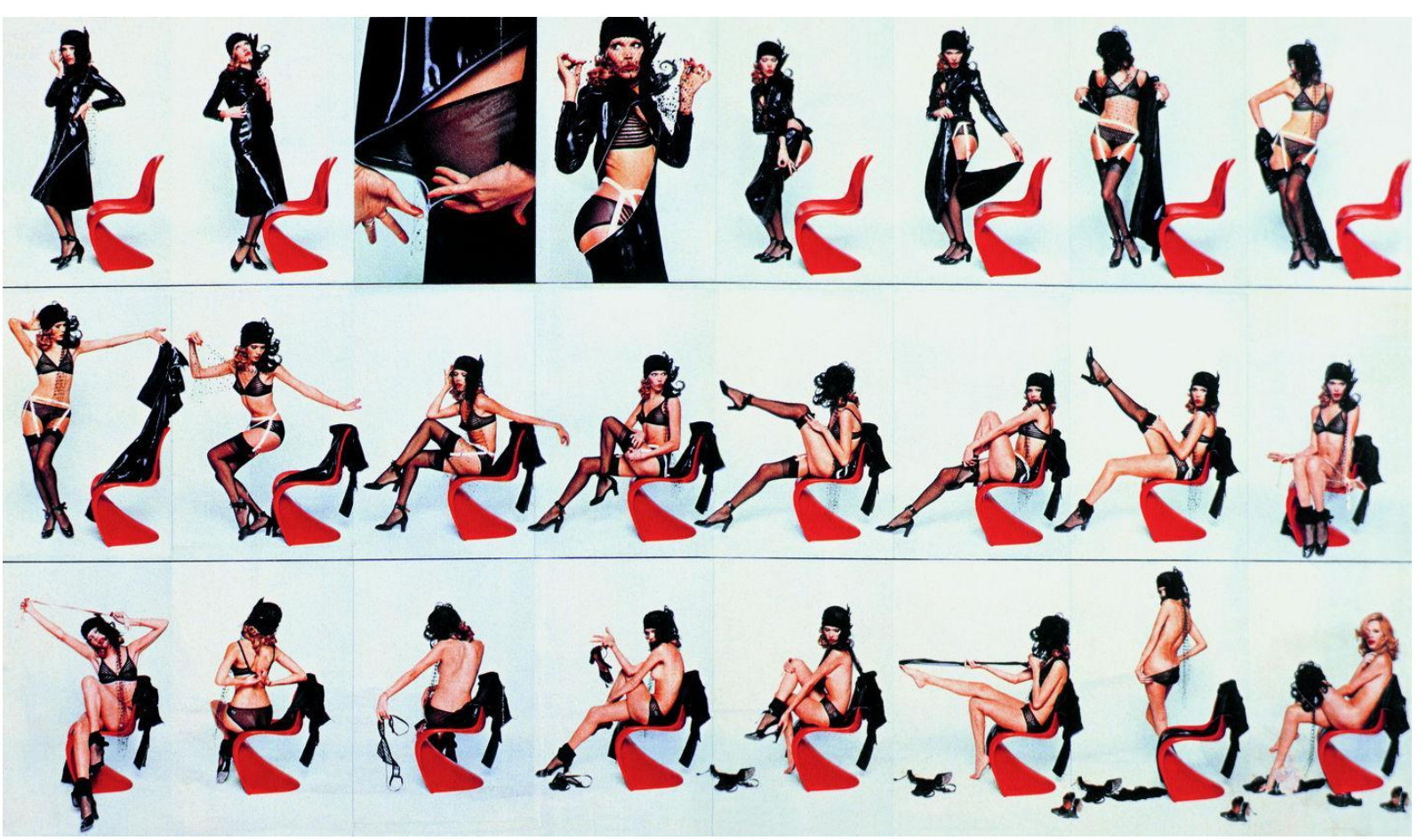

Fig. 5. Secuencia fotográfica "Cómo desnudarse delante de su marido" (How to undress in front of your husband) con la silla Panton, 1970. Publicada en la revista Nova en 1971.

Fuente: Vitra. https://www.vitra.com/en-us/magazine/details/sitting-sexy.

\section{Diseñadoras: pioneras e invisibles}

¿Por qué no hay presencia de arquitectas diseñadoras en espacios de reconocimiento como las exposiciones, las publicaciones o los catálogos? No es porque no las hubiera, es porque han sido excluidas o relegadas a un segundo plano. A pesar de las dificultades que las mujeres tuvieron para acceder a la educación y a los medios de producción, es posible reconocer al menos una veintena durante el siglo XX, lo cual es un número significativo.

Según Ana López-Navajas la actividad de las mujeres no se recoge en el relato canónico de la historia, ni en el de las artes o las ciencias, con un marcado carácter androcéntrico, cuando se las incorpora, se hace como una excepcionalidad ${ }^{28}$. Sin embargo, aunque no consten no existe una historia sin mujeres. Reescribir la historia desde una perspectiva feminista, más justa, menos sesgada implica recuperar la memoria y el rigor en el relato histórico para construir otras genealogías.

Para esto, es necesario poner en evidencia cómo quienes construyen la historia del diseño incorporan sus prejuicios de género, clase o raza. Historiadores, críticos, comisarios, como miembros privilegiados de la comunidad y los colectivos profesionales afines, construyen imágenes y explicaciones que refuerzan sus lugares y valores culturales ${ }^{29}$.

En los ejemplos de publicaciones, exposiciones y catálogos revisados, se verifica la ausencia de diseñadoras. Nos propusimos armar una línea de tiempo 
propia que incluye al menos 20 diseñadoras que no han sido mencionadas, 20 sillas fantasmas. El ejercicio sirve para interrogarnos sobre las prácticas sociales, académicas, institucionales y comerciales que producen estas selecciones.

Algunas de las preguntas que nos hicimos son ¿Qué diseñadoras han sido expulsadas de las antologías? ¿Cuáles han sido los criterios que explican esta exclusión del canon? ¿Bajo qué condiciones culturales y materiales diseñaban estas mujeres? ¿Cuáles fueron sus estrategias de resistencia?

Hacia fines del siglo XIX, donde comienza nuestra línea de tiempo se discutía si la mujer tenía capacidades intelectuales, si debía ser admitida en las instituciones de enseñanza, si debía tener acceso a los derechos civiles. Si la mujer $^{30}$ no disponía de una fortuna familiar propia o no accedía a un matrimonio provechoso y al no tener acceso a la formación no podía mejorar por sí sola su condición económica. Se la hacía responsable del hogar, donde debía hacer de madre y esposa devota. El hogar era el espacio interior, íntimo (y femenino) que se oponía al exterior y público, donde los varones ejercían su soberanía:

“... pocas mujeres hicieron de la arquitectura su profesión, y por norma general las que lo hicieron, o fueron relegadas a un segundo plano eclipsadas por los reconocidos nombres de sus compañeros o mentores, o concentraron su labor en tareas consideradas más propiamente femeninas por su vinculación al mundo doméstico, tal como el diseño de interiores o de mobiliario" 31.

El diseño de muebles así como otras facetas de trabajo en la arquitectura, como el diseño de interiores, textiles o utensilios, formaron parte de la alteridad ${ }^{32}$ asignada al rol femenino. Esta característica de otredad ${ }^{33}$ se manifestó en las desigualdades de género y la asignación de roles, que a su vez determinaron en el ejercicio de la profesión "tareas propias de mujeres y otras que son propias de varones" 34 . Las mujeres ocuparon aquellos espacios y tareas de soporte, de menor jerarquía e importancia, más rutinarias o consideradas menos competitivas.

Del sesgo androcéntrico de quienes han narrado la historia de la arquitectura, valorado desde su propia norma y experiencia, surge un reconocimiento desigual de las aportaciones de hombres y mujeres. Así, los diseños de sillas adquieren relevancia cuando son de autoría masculina elevando el estatus de la profesión y operan de manera contraria en el caso de las mujeres arquitectas. 


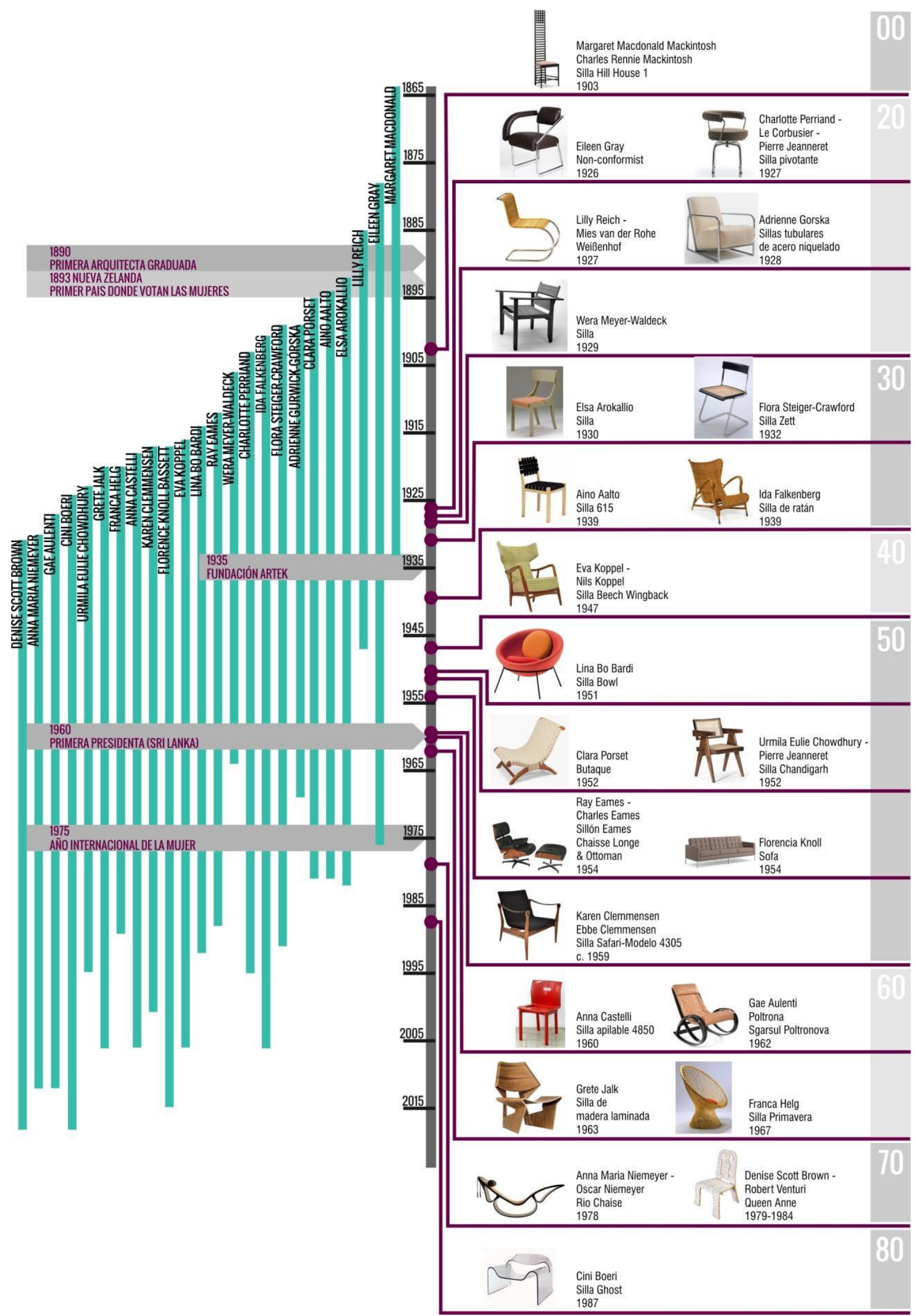

Fig. 6. Línea de tiempo que organiza piezas de mobiliario ausentes en las antologías y sus respectivas diseñadoras. Elaboración de Florencia Marciani, enero 2017. 


\section{El trayecto de formación}

Estas diseñadoras provienen o estuvieron vinculadas a instituciones que fueron espacios propicios para su desarrollo profesional y artístico. La generación de pioneras no se entiende sin los espacios académicos que las habilitaron. Si bien es cierto que el acceso a la educación era minoritario, aquellas que estudiaron lo hicieron con la convicción de ejercer y destacar. Estas mujeres abrazaron la modernidad, tenían ganas de aprender, salir al campo laboral y de recrearse. Las mujeres arquitectas se situaron entre las primeras protagonistas de este cambio sociocultural sin precedentes.

Eileen Gray estudió en la Slade School of Fine Art de Londres, fundada en 1871, institución que desde el primer momento recibió alumnas mujeres. También la Escuela de Artes de Glasgow donde se formó Margaret MacDonald, estudiando trabajo de metal y textiles, fue un sitio propicio para las mujeres en un momento en el que las escuelas de arte sólo admitía a varones. Allí se contrataban profesoras, se hacían exhibiciones de las obras de artistas mujeres y las estudiantes participaron activamente del movimiento sufragista.

Lilly Reich, desarrolló sus primeros trabajos en Viena en 1908, en los talleres de diseño, Wiener Werkstätte, trabajando en el estudio de Josef Hoffmann con quien desarrolló el sillón y sofá Kubus. Volvió a Alemania en 1911, al año siguiente se incorporó a la Deutscher Werkbund y en 1918 se graduó en arquitectura. Elsa Arokallio y Aino Marsio Aalto están entre las primeras arquitectas de Finlandia, país que fue el pionero en abrir esta carrera para las mujeres.

Clara Porset tuvo una educación poco común para las mujeres de su generación, más aún en el ámbito de influencia hispánica. Realizó sus estudios secundarios en Nueva York, a donde regresaría en 1925 para realizar el bachillerato en Artes de la Universidad de Columbia. Entre 1928 y 1931 estudió arquitectura y diseño de muebles con el arquitecto Henri Rapin en París, haciendo cursos de historia de arquitectura y de arte, así como de teoría de la arquitectura en la École de Beaux Arts, de Estética en la Sorbonne y cursos de Arte en el Museo del Louvre. Se interesó por las teorías y prácticas de la Bauhaus, por lo que buscó en el Black Mountain College, en Carolina del Norte, Estados Unidos a Josef Albers con quien estudió diseño básico y de muebles en 1934.

Es importante el acceso que tuvieron estas diseñadoras a las escuelas de artes y oficios. Muchas de ellas manejaron profundamente técnicas y materiales específicos para el diseño artesanal e industrial.

Wera Meyer-Waldeck, después de su formación como maestra de jardín de infancia, realizó estudios gráficos en la Academia de Artes Aplicadas de Dresde. En 1927 ingresó a la Bauhaus de Dessau y mientras estudiaba arquitectura y pintura, tomó lecciones de carpintería en la escuela. En 1932 se convirtió en la primera mujer en rendir examen de carpintería en el estado federado de Turingia. Aino Marsio, siendo estudiante, trabajó como aprendiz de carpintería y 
albañilería, experiencia que influyó de manera notable en su desempeño profesional.

Grete Jalk había sido aprendiz de ebanista y estudió mobiliario en la Real Academia de Bellas Artes de Copenhague, antes de establecer su propia oficina de diseño. En 1947 realizó uno de sus primeros proyectos como diseñadora independiente, un conjunto de muebles para un "refugio de mujeres autónomas".
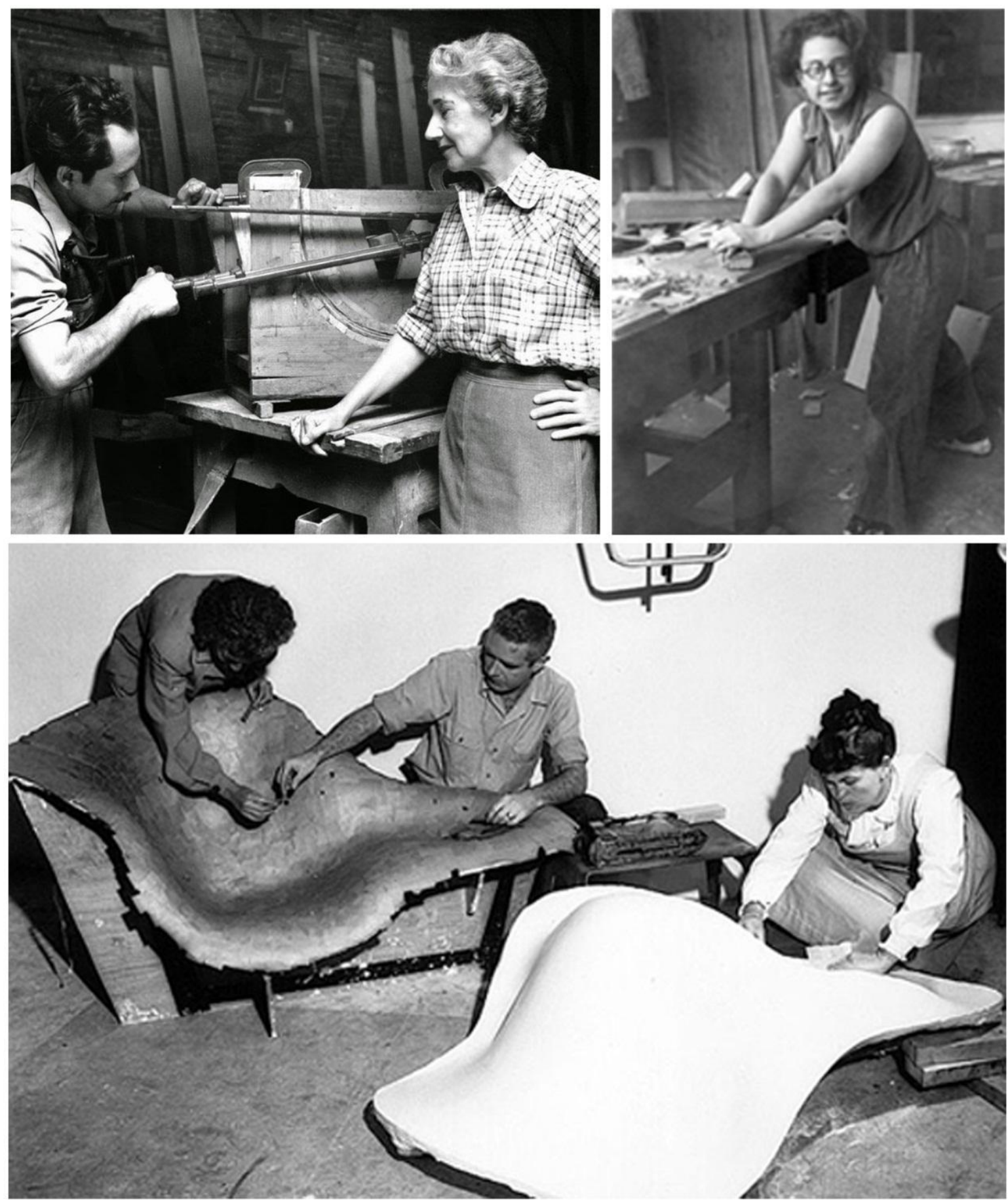

Fig. 7. Trabajo en taller. Clara Porset y el jefe de producción de su taller 1951-1952 - Wera Meyer-Waldeck en la carpintería de la Bauhaus de Dessau, Foto: Gertrud Arndt, 1930.

Bauhaus Archive - Ray Eames en el taller, 1948, Fuente: Eames Office LLC. 


\section{Ruptura de los estereotipos}

A pesar de la recurrente asociación de la mujer con el hogar y por lo tanto con el diseño de los interiores de la casa, encontramos que estas diseñadoras viajaron mucho. Buena parte de estos desplazamientos eran específicos viajes de estudio donde conscientemente aprovechan para conocer ciudades, edificios, arquitectos, modos de vida, etc. El viaje les permitió entender lo relativo de las normas y distanciarse de roles tradicionales.

Es sorprendente conocer los recorridos que realizaron. Wera MeyerWaldeck, había vivido en Egipto y en Suiza, Urmila Chowdhury en India, Japón, Australia y Estados Unidos. Aino Marsio viajó con dos de sus compañeras de estudio por Alemania, Suiza e Italia antes de graduarse y posteriormente también con Alvar Aalto. Elsa Arokallio viajó a Marruecos. Flora SteigerCrawford, nació en la India y se formó en Suiza. Eileen Gray, viajó con su padre que era pintor por Italia y Alemania, posteriormente vivió en París. Viajó por África y por América del Norte. Charlotte Perriand recorrió Brasil, Congo, Inglaterra, Francia, Japón, Nueva Guinea, Suiza, y Vietnam. Florence Knoll, pasaba sus vacaciones en Finlandia donde tomó contacto con el diseño escandinavo. No son "mujeres de su casa", son personas cosmopolitas, curiosas y desafiantes.

Por eso no sorprende que diseñadoras como Eileen Gray, Charlotte Perriand y Lilly Reich fueran piezas claves para equipar los interiores de las modernas viviendas que realizaban reconocidos arquitectos. En el ático que Le Corbusier diseñara para Charles de Beistegui en 1929, los muebles son de estilo clásico y de factura artesanal. Para ese entonces Eileen Gray ya había diseñado varias de las piezas icónicas del mobiliario moderno. La silla Non conformist (1926) que prescinde de un antebrazo para liberar el movimiento del cuerpo y permitir girar sin restricciones, es una alegoría que materializa los ideales de libertad y vanguardia de estas diseñadoras.

Otro rol que cumplieron estas diseñadoras fue el de dirigir empresas de diseño. En 1935 los Aalto, junto con Maire Gullichsen, amiga y principal cliente, y Nils-Gustav Hahlin fundaron Artek (Arte+Técnica), una empresa dedicada a la "industria doméstica" que vendía accesorios de iluminación y muebles diseñados por Alvar y Aino. Desde su creación Marsio se desempeñó como directora creativa y a partir de 1941 se convirtió en su directora general hasta su muerte en 1949. El diseño del stand de Artek que realizó para la Trienal de Milán de 1936 recibió el Grand Prix.

La multipremiada arquitecta italiana Anna Castelli colaboró con Kartell desde 1966, empresa precursora de la producción de objetos de plástico de uso cotidiano en Italia .

Florence Knoll, considerada una pionera del diseño moderno norteamericano, fue líder de la empresa de equipamientos Knoll. Aun desde su posición de privilegio, sus obras no aparecen en las antologías. En 1961 fue la primera mujer en ganar la Medalla de Oro por Diseño Industrial entregado por el Instituto Americano de Arquitectura (AIA). En 2003 fue reconocida con la 
Medalla Nacional de las Artes, y en 2004 se realizó una exposición de su propio trabajo para el Museo de Arte de Philadelphia. Phillip Hofstra expresa en el libro que le dedica a: "Florence Knoll podría haber continuado el trabajo de arquitectura, pero las oportunidades eran mayores para las mujeres en el diseño de interiores" 35 .

La actitud inconformista de las diseñadoras las llevó a producir diseños que se alejaban del lenguaje canónico. Mientras que el mainstream (¿o manstream?) hablaba de la racionalidad, de la industrialización y de las máquinas de habitar, Eileen Gray se preocupaba por la humanización, de "las más íntimas necesidades de la vida subjetiva" 36 Perriand explicó cómo su diseño buscaba "humanizar" la frialdad de las sillas modernas, abrazando con el cojín circular el cuerpo de la persona sentada. El manifiesto de la empresa Artek, hablaba de darle una perspectiva humana al modernismo y se interesaba por los materiales naturales. Lo hacen reinventando el uso de la madera, el cuero y las fibras naturales. Tal es el caso de las sillas diseñadas por Lina Bo Bardi, Clara Porset, Urmila Eulie Chodhury, Eva Koppel, Karen Clemmensen, Ray Eames, y otras.

Desde su compromiso social, Ida Falkenberg señalaba que los muebles de tubos de acero que eran una solución recurrente en los interiores modernos, eran costosos para la clase trabajadora, e incorporaba sus diseños de ratán en sus casas modelo, combinándolos además con mobiliario tradicional.

Franca Helg trabajó para la firma Bonacina y seguía de cerca cada instancia del proceso llevado a cabo por artesanos del oficio del mimbre. Le interesaba especialmente articular las técnicas tradicionales de trabajo del material con las nuevas posibilidades de expresión y manipulación del mismo.

Cabe preguntarse si quizá por esto no son incluidas en las selecciones. Si miramos la línea de tiempo vemos un paisaje diferente al de las sillas cromadas con cuero negro que se ven en las antologías hegemónicas. Es probable que uno de los motivos para la exclusión sea éste, aunque no el único. El diseño de asientos y de mobiliario en general, tuvo su auge con el desarrollo de nuevos materiales y procesos de producción que afianzados en los conceptos de progreso y mecanización, se alejaron de diseños más enraizados en lo humano y artesanal, tanto en materiales como en procesos de fabricación.

\section{Invisibilización}

Margaret MacDonald junto a su marido Charles Rennie Mackintosh realizaron diseños de interiores que renovaron el lenguaje de la arquitectura. Era reconocida en su época, sin embargo hoy se recuerda a su socio como el autor de estas obras. Él decía de ella que era un genio y que sus aportes no eran la mitad sino tres cuartos del trabajo. El respaldo de la silla Mackintosh se basa en los diseños verticales y estilizados de los dibujos de MacDonald. El catálogo de Cassina que la produce actualmente, como la colección de miniaturas de Vitra indican a Mackintosh como su único autor. ${ }^{37}$ En la publicación de la exposición 100 sillas clásicas de Vitra, se la menciona como de gran influencia aunque no se 
reconoce su autoría. El concepto de musa es otro mecanismo de discriminación que subordina a estas diseñadoras a la figura masculina. También los integrantes de un equipo quedan sometidos a un único individuo; Penny Spark se refiere a Charles Rennie Mackintosh como "el arquitecto escocés que trabajó como parte de un grupo conocido como el Glasgow Four" 38 . No obstante la escritora reconoce en su libro The modern interior ${ }^{39}$ las aportaciones de Margaret McDonald.

El caso de Lilly Reich es particularmente significativo. La arquitecta alemana que trabajó con Ludwig Mies van der Rohe, es conocida a raíz de este hecho, pero al mismo tiempo, se desconocen sus aportaciones ya que muchas son atribuidas al arquitecto. Según Albert Pheiffer, Vice Presidente de Diseño y Dirección de Knoll, investigador de la obra de Lilly Reich:

“...se está convirtiendo en más que una coincidencia que la involucración y éxito de Mies en el diseño de exposiciones comience al mismo tiempo que su relación personal con Reich. Es interesante resaltar que Mies no ha desarrollado ningún mueble moderno de manera exitosa ni antes ni después de su colaboración con Reich" ${ }^{40}$.

En 1929 como directora artística de la representación alemana en la Exposición Internacional de Barcelona, participó junto a Mies del diseño del Pabellón Nacional, donde por primera vez se exhibió la silla Barcelona, y que poco a poco comienzan a ser reconocidos, pabellón y silla, como de doble autoría tanto de Mies como de Reich. La colección de Vitra ignora a Reich y Sparke se refiere a Mies Van der Rohe como "... muy conocido por sus muebles de acero y cuero, especialmente su silla de Barcelona de 1929."

Wera Meyer Waldeck, produjo, como alumna de la Bauhaus varias piezas como la silla de 1929 que incluimos en la línea de tiempo. Hannes Meyer, con quien compartía el apellido pero no parentesco, la contrató ese mismo año para trabajar en el proyecto de la Escuela de Sindicatos de Bernau y reconoció su labor en una carta:

"todos los encargos han sido realizados con celo, profesionalidad y con una especial implicación de su parte. A consecuencia de su preparación en el taller de carpintería, ella muestra especial interés por las cuestiones técnicas en el diseño de muebles y espacios interiores"41.

El escritorio que diseñó para dicha escuela, hoy es asignado a Hannes Meyer en algunos sitios de Internet, si bien los tres archivos oficiales de la Bauhaus reconocen la autoría a Wera Meyer Waldeck.

Charlotte Perriand colaboró desde 1927 con el estudio de Le CorbusierJeanneret, diseñando los interiores de las más emblemáticas obras del siglo XX como la Ville Savoie y la Unidad de Habitación de Marsella. La silla Pivotante (1927) también llamada $L C 7$ fue diseñada por la arquitecta para su propio 
apartamento y presentada en Salon d'Automme de 1928. A partir de allí es que Le Corbusier decide contratarla. La sigla $L C$ no ayuda a clarificar la verdadera autoría de la silla, pero posiblemente le aumenta valor de mercado.

Charlotte Perriand hizo moderno a Le Corbusier en el mobiliario. Su primera colaboración fue la renovación del interior de la casa La RocheJeanneret. Entre los aportes y contribuciones de Perriand a esta vivienda están la rampa en voladizo, la mesa de alto brillo y ligeras patas cromadas, así como el mueble ubicado bajo la rampa También sustituyó los sillones Thonet que había incorporado Le Corbusier por unos más modernos diseñados por ella misma ${ }^{42}$.

"En lo que se refiere a su diseño, es más conocido por su silla Grand Confort y su Chaise Longue en la que trabajó con su asistente, Charlotte Perriand.” señala Penny Sparke mencionando a Le Corbusier. ${ }^{43}$

Aún hoy se le siguen negando a Charlotte Perriand sus propios diseños, siguen apareciendo en numerosas citas como de Le Corbusier y al igual que hiciera con la apropiación de la casa de Eileen Gray, también exhibió esta silla en una muestra sobre "equipamiento doméstico".

En una entrevista realizada por Martín Meade y Charlotte Ellis, Perriand explica lo que sucedió con los créditos de la silla $L C 7$ :

"Es simple: Madame Weber conoció a Le Corbusier, fue una gran admiradora de su pintura y escultura y fundó el museo de Le Corbusier en Suiza. Quería producir una nueva edición de las sillas de 1929 y fue ella quien arregló la nueva edición con Cassina. Desde el punto de vista comercial, el nombre "Le Corbusier" suena -los tres nombres no tienen la misma resonancia- pero las etiquetas de la edición de Cassina dicen "Creación 1929, Le Corbusier - Jeanneret Perriand”. Se hicieron cambios (la edición de 1929 tenía relleno de plumas y la edición de Cassina tiene espuma) pero en todos los aspectos importantes, la edición de Cassina es idéntica a la edición de 1929. Todavía conservo el control de los diseños, a través de la Fundación Le Corbusier"44.

En la actualidad la empresa Cassina la produce como Charlotte Perriand, aunque en el catálogo de Vitra continúa apareciendo como "Le Corbusier Jeanneret - Perriand".

Urmila Eulie Chowdhury fue una de las primeras arquitectas calificadas de Asia. Nacida en la India, se graduó en Japón y regresó a la India en 1951, incorporándose al equipo encargado del diseño y construcción de la ciudad de Chandigarh. Además de tener a su cargo el diseño y construcción del Home Science College, el Women's Polytechnic y varias casas para los Ministros del Gobierno, diseñó el mobiliario para sus proyectos y para los de Pierre Jeanneret ${ }^{45}$. Estas son las famosas sillas de teca, árbol propio de la India, y esterilla, que están presentes en los grandes edificios de Chandigarh. 
En 1954 Chowdhury fue premiada con la medalla de oro por el Presidente de su país por el diseño de muebles económicos. En un artículo de Sumit Kaur, arquitecta Jefe de Chandigarh dijo: "Todos los muebles del complejo del Capitolio y de la Universidad Panjab, el auditorio, la biblioteca y la casa de huéspedes, fueron diseñados por ella" 46 .

La muestra Le Corbusier \& Pierre Jeanneret, Low Cost Furniture and Other Items from Chandigarh celebrada en Ginebra en 2011 expuso los muebles diseñados por Chowdhury pero les fueron asignados a Jeanneret con el objetivo de brindar "una visión más precisa de la obra humanista de Pierre Jeanneret, a menudo ensombrecida por su ilustre primo".

Aino Marsio trabajó junto a su marido Alvar Aalto y muy poco se ha dicho de ella, aunque su trabajo junto con su reconocido compañero cada vez se hace más evidente. Los diseños producidos en la oficina estaban usualmente firmados como "Aino y Alvar Aalto". La hija de ambos Johanna, expresaba lo siguiente:

"En general, Alvar no tenía tiempo para diseñar muebles, porque estaba concentrado sobre las obras arquitectónicas de la oficina. El aporte de Aino no consta sólo de los muebles, sino también de proyectos de interior. Además de todo eso, Aino dedicaba la mayoría de su tiempo a Artek" ${ }^{47}$.

Inspirada en la producción artesanal finlandesa Marsio crea numerosos bancos, sillas, butacas y sillones en materiales como el abedul y tejidos de algodón. La silla 615 es un ejemplo de estas exploraciones. El Sillón en voladizo (1932), uno de los primeros muebles de madera laminada, fue diseñado por Aino y Alvar ${ }^{48}$.

Estos aportes no se reflejan en las atribuciones de las autorías en las bibliografías ni en los museos. Tanto el MoMA como el Met atribuyen solo a Alvar las diferentes sillas diseñadas por el equipo ${ }^{49}$.

Entre las exposiciones organizadas por fundaciones dedicadas a "los maestros", encontramos la exposición de Alvar Aalto en Caixa Forum Madrid en 2015, organizada por Vitra Design Museum y Alvar Aalto Museum, en colaboración con la Obra Social "la Caixa". La misma omite desde la presentación y notas de prensa las figuras de Aino Marsio y Elsa Kaisa Mäkiniemi. Por el contrario en 2016 se expuso en la Bard Graduate Center Gallery de Nueva York la primera exposición en los Estados Unidos sobre Artek, Artek and the Aaltos: Creating a Modern World. Esta muestra fue el resultado de la investigación de la directora de la galería Nina Stritzler-Levine, y por vez primera se le da un espacio en igualdad de condiciones a la figura de Aino Marsio así como a los demás fundadores de la empresa, Nils-Gustav Hahl y Maire Gullichsen ${ }^{50}$.

La diseñadora y pintora Ray Eames (1912-1988) fue cofundadora del American Abstract Artists Group. Junto a Charles Eames conformaron uno de los equipos de diseñadores más reconocidos y mediáticos del siglo XX principalmente en los años de posguerra. Ella fue la que aportó el lenguaje orgánico distintivo de 
la producción de esta sociedad. A pesar de su trabajo conjunto, su labor no ha sido reconocida de la misma manera que la de su socio. La muestra de 1946 en el MoMA se llamó Furniture designed by Charles Eames ${ }^{51}$.

La Chaise Longue \& Ottoman de 1954 que hoy está presente en exposiciones y museos, resume las investigaciones de Ray Eames en contrachapado. No obstante la publicidad de Hermann Miller siempre presentó la pieza como diseñada únicamente por Charles Eames. En 1956 fue lanzada en televisión y la conductora presentó a Charles como "este inteligente diseñador", posteriormente ingresó Ray que fue recibida con la siguiente frase: "Esta es la señora Eames y ella nos va a contar cómo ayuda a Charles a diseñar estas sillas". De la misma manera Sparke se refiere a ella desde la figura del arquitecto, como “...su esposa Ray, que trabajó en colaboración con él desde los años cuarenta en adelante, en el cine experimental".

En 1967 la British Society of Artists and Designers premió a Charles Eames. A Ray Eames le entregaron una rosa roja ${ }^{52}$. Después de la muerte de él en 1978, la diseñadora se dedicó a catalogar su trabajo creativo y fue a partir de entonces cuando comenzó a aparecer en los créditos. En 1985 en una ceremonia póstuma en la World Design Conference se premia a Charles como el "Diseñador más influyente del mundo", en la World Design Conference. Ray solo asiste a la ceremonia. En una entrevista realizada por Pat Kirkhan se produce el siguiente diálogo:

"R.E. "Me molesta cuando la gente escribe que Ray "hizo el color". En primer lugar, está mal. Los dos lo hicimos. Pero también implica que lo hice y nada más. De la misma manera dicen 'Oh, Ray ella hizo el interior' porque sienten que el exterior era todo de Charles.

P.K.: ¿Crees que es porque eres una mujer y las mujeres están asociadas con la decoración de interiores?

R.E.: Por supuesto"53.

Franca Helg trabajó junto a Franco Albini en diseños especiales para diferentes firmas: objetos de Brionvega, Cassina, Arflex, Arteluce y Poggi. Para Vittorio Bonacina, empresa dedicada al trabajo del mimbre, Helg diseñó y construyó planos de despiece y morfología de equipamiento a escala 1:1 que fueron utilizados como matrices para el moldeo y conformado en curva de las diferentes piezas.

Si bien en documentos de la Fondazione Franco Albini se señala la autoría compartida de la silla Primavera, Mario Bonacina dice en una entrevista:

"Después de diseñar varias piezas para nosotros, las cuales aparecían siempre en los catálogos y revistas con el nombre de Franco Albini y Franca Helg, ella les preguntó a mis padres si podía firmar las piezas con sólo su nombre ya que las había diseñado ella sola. No 
habían salido de las manos de Franco Albini estos productos. Obviamente ella lo preguntó con mucha delicadeza, diciendo, sé el nombre de Albini es más importante que el mío y no quisiera esto les cree problemas. Por otro lado, para mis padres no era ningún problema porque lo importante era el diseño y sus proyectos eran muy bellos y diferentes de los de Albini y era claro que tenían un toque, una sensibilidad distinta en la realización de estas piezas" 54 .

La colección de 9 Sillas de Denise Scott Brown y Robert Venturi fue creada para hogares de recursos limitados55: "Resultó que Knoll no iba a usarlas para sentar a los pobres. Las estaban usando como arquitectura de estrellas" 56 .

En ese momento, todo el crédito por las sillas se le dio a Venturi y, a pesar de las solicitudes de Scott Brown, Knoll todavía no reconoce su contribución al proyecto ${ }^{57}$. Un tema recurrente para la arquitecta como el caso del Premio Pritzker entregado solamente a su socio en 1991. Denise Scott Brown lo recalca en esta entrevista:

"Le agradecería que recuerde que he trabajado con Bob desde 1960, dibujando primero y luego en la práctica. Durante mucho, mucho tiempo, ha sido trabajo de ambos, aunque nadie lo aceptaría. (...) Así que estoy teniendo que luchar esa batalla también. No pusieron mi nombre en las sillas Knoll, pero saben muy bien cuál era mi papel en todo eso" 58 .

La arquitecta participó en el diseño de la silla Queen Anne desde su conceptualización hasta el diseño de detalles. El MoMA tiene una en su colección y cita correctamente a ambos.

Como vemos, estos procesos de invisibilización se dan especialmente en las instancias en que las diseñadoras trabajan con diseñadores reconocidos que muchas veces son sus parejas, padres o hermanos. Las mujeres quedan a la sombra de sus compañeros masculinos, porque existe la presunción histórica y consolidada desde la comunidad, críticos, académicos, etc., de que no son capaces de crear en igualdad de condiciones.

Así también el caso de Anna María Niemeyer (1930-2012), hija única del famoso Oscar Niemeyer, trabajó con su padre en la construcción de Brasilia, después de haber sido responsable de la arquitectura interior de varios edificios públicos en la ciudad. En los años setenta, crearon una línea de muebles, expuesta en diferentes instituciones en Brasil y en el extranjero. En 1979, fueron expuestos en el Museo Pompidou como de la sola autoría del arquitecto ${ }^{59}$.

Se evidencia también en el trabajo prácticamente desconocido de Elsa Arokallio, pionera finlandesa, que diseñó junto con la artista textil Marja Kansanen los interiores de algunas salas del Parlamento de su país, —obra del arquitecto Johan Sigfrid Sirén-, en donde se buscó mostrar las últimas tendencias del diseño finlandés para fortalecer la imagen pública del gobierno. 
Aunque algunas publicaciones atribuyen a Sirén la autoría de la totalidad del diseño, él diseñó las salas principales y delegó las secundarias en un grupo de diseñadores de su generación, que trabajó con artesanos sumamente calificados y con materiales de gran calidad. La silla de abedul que incluimos forma parte del diseño para la Habitación Roja.

Otras sillas que no aparecen en las colecciones analizadas son sillas que ganaron premios y que fueron reconocidas por su calidad en su momento. Muchas de estas piezas originales son rematadas hoy en importantes subastas por lo que no habría razón comercial para no incluirlas en un catálogo de souvenirs. Es decir, ejemplos exitosos pero invisibles para las antologías.

Por ejemplo la silla de metal apilable que Flora Steiger Crawford diseñó para la Zett Haus en Zurich (1932). Tanto la silla Zett, como otros diseños de Steiger-Crawford, actualmente son producidos y comercializados por firmas internacionales. Elegantes, funcionales y con la posibilidad de ser producidos en serie, sus diseños forman parte del escalafón más alto del diseño del Movimiento Moderno. Sin embargo, no aparecen en las colecciones.

La poltrona en mimbre y ratán realizada en 1939 por Ida Falkenberg, una de las arquitectas más importantes del movimiento moderno en Holanda, ganó el primer premio de un concurso de Wohnbedarf de Zurich. Esta empresa fue fundada por Werner Max Moser, Sigfried Giedion y Rudolf Graber. Sus muebles se comercializaban en Metz \& Co empresa líder en muebles para el hogar y diseño moderno en los Países Bajos.

Clara Porset diseñó los muebles para proyectos de viviendas de, entre otros arquitectos mexicanos, Luis Barragán y Mario Pani. En 1941 participó del concurso Organic Design for Home Furnishing organizado por el MOMA de Nueva York ganando uno de los premios.

La arquitecta noruega Eva Koppel y su socio Nils Koppel recibieron la Medalla Eckersberg, otorgada por la Real Academia Danesa de Bellas Artes juntos en 1955. Igual situación se plantea con la Silla Safari de los arquitectos daneses Karen y Ebbe Clemmensen, un clásico del mobiliario escandinavo de mediados del s. XX. Por ella obtuvieron en 1948 el Premio Knud V. Engelhardt. La italiana Anna Castelli diseña la Silla superponible 4870 que fue la ganadora del Compasso d'Oro en 1987.

Si bien la silla Bowl fue un autoencargo y pertenece a la primera fase de la carrera de Lina Bo Bardi en Brasil, ya en 1953 la revista American Magazine Interiors le dedica un artículo. Así como la Sgarsulv de Gae Aulenti para Poltronova, fue ampliamente difundida en los medios. En 2012, Aulenti recibió la Medalla de Oro de la Triennale di Milano por su trayectoria artística en reconocimiento a su posición como uno de los maestros del diseño italiano. No hay piezas de ella en las colecciones.

Grete Jalk gana con sus sillas de madera laminada el premio de un concurso organizado por el Daily Mail en 1963. Ha sido incorporada con posterioridad a la colección de Vitra y está en la colección del MoMA. 

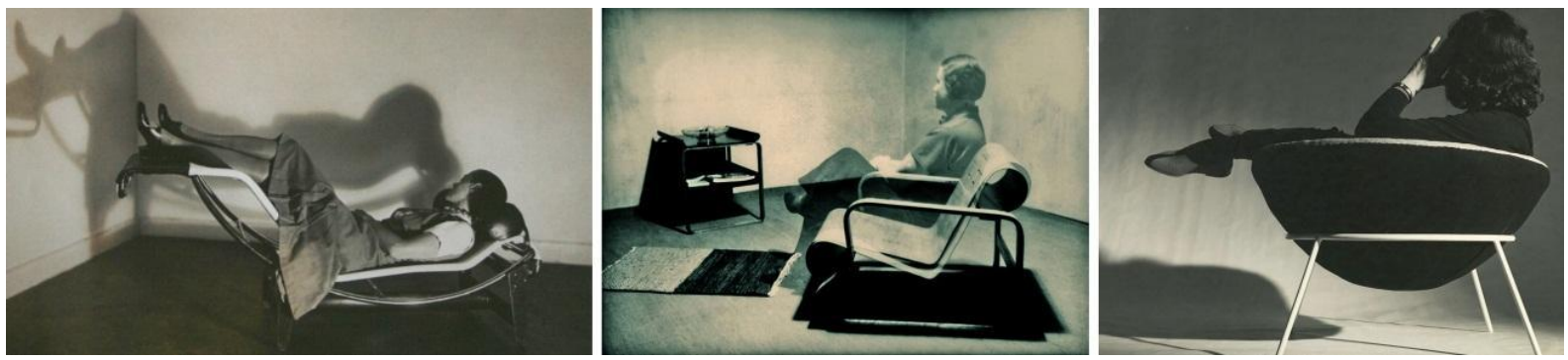

Fig. 8. Invisibilidad. Charlotte Perriand en la Chaise Longue, Aino Aalto en la silla Paimio, fotomontaje, década 1930. Alvar Aalto Museum, Colección Artek VG Bild-Kunst, Bonn, 2014 - Lina Bo Bardi en la silla Bowl. Instituto Lina Bo e P. M. Bardi.

\section{7. ¿Para qué sirve una antología?}

Las antologías son cuerpos de conocimiento constituidos que otorgan lugares de poder a unos y no a otros. La construcción de una antología supone un proceso de selección de obras que se consideran mejores a partir de una ideología, postura estética, corriente, etc.

Estas antologías se constituyen en tecnologías de género ${ }^{60} \mathrm{y}$, como tales, tienen el poder para controlar el campo del significado social y entonces producir, promover e "implantar" representaciones de género.

A partir de nuestro estudio encontramos diseñadoras expulsadas del canon, tanto en catálogos de productos como en una publicación con fines pedagógicos.

Sus obras constituyen una lista o elenco de piezas consideradas valiosas, y dignas por ello de ser estudiadas y comentadas. Incorporar las sillas que cumplen con los requisitos formales esperados es una primera acción, a sabiendas de que los estándares estéticos también han sido consolidados por consensos que dejaron fuera los conocimientos y experiencia de las mujeres. Pero alcanza solamente con sumar producción de las mujeres al canon preexistente. Se lo debe cuestionar y deconstruir, porque estas listas implican, en el fondo, una definición de la profesión. Virginia Woolf, lúcidamente, señala en Una habitación propia: "pensé en lo desagradable que era que le dejaran a uno fuera; y pensé que quizá era peor que le encerraran a uno dentro".

La última silla que proponemos para nuestra línea de tiempo es la Ghost Chair de Cini Boeri y Tomu Katayanagi ganadora del Compasso d'Oro en 1979. Nunca mejor elegido el nombre para la pieza diseñada por la arquitecta que en los años 50 abría su propio estudio, enfrentando a sus colegas masculinos que le señalaban que la arquitectura no era para mujeres:

"Esta silla, llamada fantasma, fue proyectada por mí, en un período en que estaba tremendamente harta de diseñar sillas y sillones, pero era lo que todo el mundo me pedía hacer... Así que me dije, hagamos una que no se vea."61 
¿Qué ocurre con las piezas producidas por mujeres que han desaparecido de la historia o que aparecen como realizadas por colegas masculinos? Críticos, comisarios y empresarios realizan selecciones sesgadas para mostrar lo que a su juicio tiene valor. Eligen lo que apoya su punto de vista y cuando su punto de vista coincide con el objeto atribuyen su creación a un hombre.

Explica la crítica literaria Judith Fetterly, en base a una revisión de la literatura escrita por mujeres en el siglo XIX y el canon norteamericano, que hay evidencia de que los varones son "lectores resistentes"62 de la ficción de las mujeres. Denise Scott-Brown, se refiere a esta resistencia cuando habla de Lord Palumbo, jurado del premio Pritzker “... él es un asno, él realmente lo es. Él dijo: "No se puede reescribir la historia" 63 . ¿El personaje desconoce que la historia se reescribe? ¿O no quiere o no siente necesidad de hacerlo porque así sostiene su posición de privilegio?

La historia, y la historia del diseño, está construida y narrada por hombres, excluyendo a la mitad del mundo: la experiencia y la perspectiva de las mujeres. Las 20 sillas fantasmas nos ponen en alerta sobre esta situación. Es necesario reconstruir las historias, generar un corpus propio y compilar. Es prioritario visibilizar y publicar porque lo que no está publicado no existe. El cambio de paradigma llegará una vez que las mujeres tengan un reconocimiento justo de sus logros y que ocupen en igualdad de condiciones espacios de decisión.

\section{NOTAS}

\footnotetext{
${ }^{1}$ En reseña del libro de CRANZ, Galen, The Chair: Rethinking Culture, Body, and Design, New York, W. W. Norton \& Company, 1998.

${ }^{2}$ Para mencionar solo dos referencias importantes en este sentido citamos al movimiento de Herstory en Estados Unidos liderado por MORGAN, Robin cuya antología Sisterhood is Powerful publicado en 1970 es considerado por la Biblioteca Pública de Nueva York como uno de los 100 libros más influyentes del siglo XX. La segunda referencia es la contundente Historia de las Mujeres en Occidente, una colección de 5 tomos dirigida por DUBY Georges y PERROT Michelle, Madrid, Taurus Ediciones, 1990-1991.

${ }^{3}$ Los temas sobre la diferencia sexual y la otredad han sido tratados por relevantes teóricos y teóricas en distintos campos del conocimiento como Marcela Lagarde, Rossi Braidotti, Luce Irigaray, Gayatri Chakravorti Spivak o Michel Foucault. En arquitectura y el arte en concreto: Katerina Ruedi, Duncan Mccorquodale y Sarah Wigglesworth (Desiring practices: Architecture, gender and the interdisciplinary, 1996); M. ${ }^{a}$ Elena Díez Jorge (Investigar sobre la arquitectura y el género: teoría y praxis de un proyecto, 2014); Griselda Pollock (Vision and Difference: feminism, femininity and the histories of art, 1991); Despina Stratigakos (Where are the Women Architects?, 2016) o Joan Rothschild (Design and Feminism: Re-visioning Spaces, Places, and Everyday Things, 1999), entre otros.

4 BLAZQUEZ GRAF, Norma, "Epistemología feminista: temas centrales" en Investigación Feminista. Epistemología, Metodología y Representaciones Sociales, México, Universidad Nacional Autónoma de México, 2010, p. 21-38.

${ }^{5}$ El material de base para este artículo se encuentra en los textos producidos por el equipo de Un día | una arquitecta, https://undiaunaarquitecta.wordpress.com/

ALVAREZ, Eva, ANNA MARIA NIEMEYER 1930-2012, 22 de junio de 2015, https://undiaunaarquitecta.wordpress.com/2015/06/22/anna-maria-niemeyer-1930-2012/ ALVAREZ, Eva, DENISE SCOTT BROWN 1931, 24 de junio de 2015, https://undiaunaarquitecta.wordpress.com/2015/06/24/denise-scott-brown-1931/

GONZALEZ GOMEZ, Heidy, ADRIENNE GURWICK-GÓRSKA 1899-1969, 17 de enero de 2017 ,
} 
https://undiaunaarquitecta2.wordpress.com/2017/01/17/adrienne-gurwick-gorska-1899-1969/

GONZALEZ GOTTDIENER, Isaura, ELSA AROKALLIO 1892-1982, 15 de septiembre de 2016 https://undiaunaarquitecta2.wordpress.com/2016/09/15/elsa-arokallio-1892-1982/

GUTIERREZ POIZAT, Sandra, URMILA EULIE CHOWDHURY 1923-1995, 8 de junio de 2015, https://undiaunaarquitecta.wordpress.com/2015/06/08/urmila-eulie-chowdhury-1923-1995/

KESMAN, Cecilia, FRANCA HELG 1920-1989, 25 de mayo de 2015, https://undiaunaarquitecta.wordpress.com/2015/05/25/franca-helg-1920-1989/

MARCIANI, Florencia, AINO AALTO 1894-1949 9 de abril de 2015

$\begin{array}{llllll}\text { https://undiaunaarquitecta.wordpress.com/2015/04/09/aino-aalto-1894-1949/ } & & & & \\ \text { MARCIANI, Florencia, } & \text { CHARLOTTE PERRIAND } & 1903-1999, & 25 & \text { de } & \text { abril de } 2015\end{array}$ https://undiaunaarquitecta.wordpress.com/2015/04/25/charlotte-perriand-1903-1999/

MARCIANI, Florencia, EILEEN GRAY 1878-1976, 31 de marzo de 2015 https://undiaunaarquitecta.wordpress.com/2015/03/31/eileen-gray-1878-1976/

MARCIANI, Florencia, GAE AULENTI 1927-2012, 13 de junio de 2015 https://undiaunaarquitecta.wordpress.com/2015/06/13/gae-aulenti-1927-2012/

MERCE, Cayetana FLORA $\quad$ STEIGER-CRAWFORD 1899-1991, 18 de abril de 2015, \begin{tabular}{llllllll} 
https://undiaunaarquitecta.wordpress.com/2015/04/18/flora-steiger-crawford-1899-1991/ & & \\
\hline MERCE, Cayetana, & FLORENCE & KNOLL & BASSETT & 1917, & 19 & de & mayo de 2015,
\end{tabular} https://undiaunaarquitecta.wordpress.com/2015/05/19/florence-knoll-bassett-1917/

MONTANER, Josep Maria, LINA $\quad$ BO BARDI 1914-1992, 13 de mayo de 2015, https://undiaunaarquitecta.wordpress.com/2015/05/13/lina-bo-bardi-1914-1992/

MUXI, Zaida, CLARA PORSET 1895-1981, 11 de abril de 2015, https://undiaunaarquitecta.wordpress.com/2015/04/11/clara-porset-1895-1981/

MUXI, Zaida, LILLY $\quad$ REICH $1885-1947, \quad 2$ de abril de 2015

\begin{tabular}{llll} 
https://undiaunaarquitecta.wordpress.com/2015/04/02/lilly-reich-1885-1947/ \\
\hline NOVELLA, Inés, KAREN & CLEMMENSEN & $1917-2001$, &
\end{tabular} de mayo de 2015, https://undiaunaarquitecta.wordpress.com/2015/05/20/karen-clemmensen-1917-2001/

OJEDA, Gueni, WERA MEYER-WALDECK 1906-1964 | HILDE WESTRÖM 1912-2013, 1 de mayo de 2015, https://undiaunaarquitecta.wordpress.com/2015/05/01/wera-meyer-waldeck-1906-1964-hilde-westrom-1912-

$\underline{2013 /}$

RODRIGUEZ BADA, Marta, ANNA CASTELLI 1918-2006, 20 de noviembre de 2016 https://undiaunaarquitecta2.wordpress.com/2016/11/30/anna-castelli-1918-2006/

ROSERO, Verónica, EVA KOPPEL 1916-2006, 28 de mayo de 2015 , https://undiaunaarquitecta.wordpress.com/2015/05/18/eva-koppel-1916-2006/

${ }^{6}$ Citado por Reinharz, Shulamit y Davidman, Lynn, "Feminist Methods" en Social Science, Oxford University Press, 1992, p. 245.

${ }^{7}$ VON VEGESACK, Alexander, DUNAS, Peter, SCHWARTZ-CLAUSS, Mathias, 100 Masterpieces from the Vitra Design Museum Collection, Vital Learning Corporation, 1996.

${ }^{8}$ MÁČEL, Otakar, WOERTMAN, Sander, VAN WIJK, Charlotte, Chairs: Catalogue of the Delft Faculty of Architecture Collection, Rotterdam, 010 Publishers, 2008.

${ }^{9}$ Se organiza de manera cronológica, poniendo claramente el acento en el "diseño de autor".

${ }^{10}$ La colección está organizada a partir de los materiales. La introducción advierte que no se encontrarán los diseños clásicos de los libros de diseño moderno o preciosas antigüedades, pero que "ilustra lo que realmente significa ser un diseñador".

11 Silla LCW (1945), Silla RAR (1948), Silla DKR (1951), Silla: No.670 y No.671 (1956) y Silla: No.684 (1958).

${ }^{12}$ La Wire Chair o DKR (1951) producida por Hermann Miller.

${ }^{13}$ Los resultados del análisis replican y amplían los conteos realizados en diccionarios de arquitectura y bases de datos presentados por el Colectivo Un día | una arquitecta en el Congreso de la Union Internationale des Femmes Architects, Washington 2015, "A Revision of the Consolidated Written History" (disponible en https://undiaunaarquitecta.wordpress.com/articulos/a-revision-of-the-consolidated-written-history/) Incluso en la bibliografía básica ocurre lo mismo. Por ejemplo en el libro de GIEDION, Sigfried, La mecanización toma el mando, Barcelona, Gustavo Gili, 1948, donde solo es mencionada Eileen Gray y donde el conteo da $97 \%$ varones y $3 \%$ mujeres. 
${ }^{14}$ Lo cual representa el 2,7\% de los personajes citados en el glosario del final del libro.

${ }^{15}$ SPARKE, Penny es autora de publicaciones como As long as it's pink: The sexual politics of taste, Virginia, Pandora, 1995, o Women's places: architecture and design 1860-1960, London, Routledge, 2003 junto a MARTIN, Brenda, publicaciones que relacionan diseño y género.

${ }^{16}$ Para el libro An Introduction to Design and Culture, 1900 to the Present Sparke utiliza como fuente la investigación realizada por Jill Seddon y Suzette Worden sobre las mujeres que trabajaron como diseñadoras en Gran Bretaña en los años de entreguerras. Women Designing: Redefining Design in Britain between the Wars, Brighton, University of Brighton, 1994. En base a este material Sparke concluye: "Si bien el enfoque de la "ocultamiento de la historia", que ha caracterizado tanto trabajo en el área de los estudios de la mujer, continúa descubriendo nombres olvidados, el panorama general es claro. El diseño en las nuevas industrias tecnológicamente orientadas estaba dominado por los hombres y el extremo más agresivamente modernista del espectro estético estaba habitado por arquitectos masculinos en su mayor parte. En términos generales, las mujeres adoptaron un modelo más conservador de la modernidad y no participaron, en gran escala, en el mundo de la práctica del diseño profesional."

${ }^{17}$ Según Carmen Espegel, a partir de 1977 (un año después de la muerte de Eileen Gray) resurge el interés por su obra y se evidencia en exposiciones a nivel internacional en Viena, Londres, Edimburgo, Nueva York, Los Ángeles, Nueva Jersey, Boston o Washington. En ESPEGEL, Carmen, Heroínas del espacio: mujeres arquitectos en el movimiento moderno, Buenos Aires, Editorial Nobuko, 2000, pp.122. No obstante, exposiciones previas a su fallecimiento en Viena (1970), Londres (RIBA en 1972-73 y Victoria and Albert Museum en 1979) o Nueva York (MoMA, 1980) son el resultado de un resurgimiento por el interés de su obra a partir de una nota de Joseph Rykwert en la revista Domus en 1968.

${ }^{18}$ BARCENILLA, Haizea, "Exponiendo historias de mujeres" en Revista Pikara, 2013, pp. 34-39.

${ }^{19}$ Le Corbusier - Charlotte Perriand. LA LUCE (Ginebra y Milán, 2015); Cuisines. 1950. Cité Radieuse, Marseille / 1930. CUBEX, Bruxelles (Bruselas, 2015); La Luce Biennale intérieur 2016 (Bélgica, 2016).

${ }^{20}$ WALLACH SCOTT, Joan, El problema de invisibilidad, México, Instituto Mora, 1992.

${ }^{21}$ Cassina, Vitra, Knoll y Artek, son empresas referentes localizadas en lugares centrales del sistema global de producción: Europa y Estados Unidos. Se especializan en el "diseño de autor" ya que sus estrategias de marketing están centradas en destacar al famoso personaje que las concibió y no tanto a otras cualidades que tiene un mueble, por ejemplo, su comodidad. Es por ello que sus catálogos están categorizados por los nombres de los creadores.

${ }^{22}$ Catálogo online de Cassina: http://www.cassina.com/en/designer (recuperado el 15 de enero de 2017).

${ }^{23}$ Ombra Tokyo, Tabouret Méribel y Tabouret Berger, LC9, LC8, LC8 OUTDOOR, LC7 y LC7 outdoor.

${ }^{24}$ Catálogo online de Artek: http://www.artek.fi/products/chairs (recuperado el 15 de enero de 2017).

${ }^{25}$ Catálogo online de Knoll: https://www.knoll.com/discover-knoll/designers (recuperado el 15 de enero de 2017).

${ }^{26}$ En este caso se transmiten varios mensajes en simultáneo: el plástico como material utilizado específicamente para tapicería, deriva en mensajes, tales como la variedad de modelos, colores y estampados, la utilidad, la durabilidad y el mantenimiento, son todos atributos que se asocian en el texto a la forma de vida contemporánea. El confort y la facilidad en las tareas domésticas se lo dedican especialmente a la mujer y a su rol que nada tiene de simbólico o implícito en este aviso; "Creado por el varón para hacérselo a las mujeres mucho más liviano", revista House \& Garden, agosto, 1949.

${ }^{27}$ Es el caso de la secuencia fotográfica con la silla de Verner Panton titulada "Cómo desnudarse delante de su marido" (How to undress in front of your husband) realizada en 1970 y publicada en la revista británica Nova en 1971. Fuente: Vitra. https://www.vitra.com/en-us/magazine/details/sitting-sexy. O la silla Ant Chair (Modelo 3107), de Arne Jacobsen fotografiada con la modelo Christine Keeler en 1963 por Lewis Morely. Fuente: Victoria and Albert Museum.

${ }^{28}$ LÓPEZ-NAVAJAS, Ana, Análisis de la ausencia de las mujeres en los manuales de la ESO: una genealogía de conocimiento ocultada, Madrid, Ministerio de Educación, 2014.

${ }^{29}$ A este respecto se refiere Diana Maffía mencionando a Londa Schiebinger (1993) sobre los padres de la ciencia moderna incorporaron sus prejuicios en las investigaciones. En MAFFÍA, Diana Helena. El vínculo crítico entre género y ciencia. Clepsydra: revista de estudios de género y teoría feminista, 2006, no 5, p. 37-58.

${ }^{30}$ Nótese el uso de la palabra "mujer" en singular para hacer referencia a como se la concebía. El uso del plural o singular no es teórica ni metodológicamente irrelevante, puesto que "la mujer" hace referencia a una esencia 
femenina única (el eterno femenino) ahistórica, de raíz a la vez biológica y metafísica. En tanto que "las mujeres" expresa la diversidad e historicidad de situaciones en que se encuentran las mujeres. No se les puede estudiar ni conceptualizar como categoría monolítica. En DE BARBIERI, Teresita, "Sobre la categoría género. Una introducción teórico-metodológica”, Revista interamericana de Sociología, 1992, vol. 6, no 2-3, p. 147-178.

${ }^{31}$ NOVAS, María, Arquitectura y género. Una reflexión teórica, Barcelona, 2014. Trabajo fin de máster para la obtención del título de Máster Universitario en Investigación Aplicada en Estudios Feministas, de Género y Ciudadanía, 2014. Universitat Jaume I. Recuperado de: http://repositori. uji. es/xmlui/handle/10234/109842.

${ }^{32}$ DE BEAUVOIR, Simone, El segundo sexo, Buenos Aires, Siglo XX, 1981.

33 "'La categoría de Otro es tan originaria como la conciencia misma", escribe Beauvoir. Pero el problema no es la Alteridad en sí misma, dado que todos somos los otros de los otros, sino que un sexo completo, con independencia de las épocas, culturas o saberes, con independencia también de las características singulares de cada una de sus componentes, haya sido continuamente designado como la Otredad absoluta de lo humano". Valcárcel, Amelia, "Beauvoir: A cincuenta años de El segundo sexo", p. 5.

${ }^{34}$ AGUDO ARROYO, Yolanda, SÁNCHEZ DE MADARIAGA, Inés, "Construyendo un lugar en la profesión: trayectorias de las arquitectas españolas" en Feminismo/s, n. 17, 2011, pp. 155-181.

35 HOFSTRA, Philip G., Florence Knoll, Design and the Modern American Office Workplace, Michigan, ProQuest, 2008.

${ }^{36}$ RAULT, Jasmin, "Fashioning Saphic Architecture" en PARKINS, Ilya y SHEEHAN, Elizabeth (eds.) Cultures of Femininity in Modern Fashion, New England, University Press of New England, 2011.

37292 HILL HOUSE 1, en Sitio de Cassina (recuperado el 15 de enero de 2017) http://www.cassina.com/en/collection/chairs/292-hill-house-1

${ }^{38}$ SPARKE, Penny, Diseño y cultura: una introducción: desde 1900 hasta la actualidad, Barcelona, Gustavo Gili, 2010.

${ }^{39}$ SPARKE, Penny, The modern interior, London, Reaktion books, 2008.

40 PHEIFFER, Albert, "Lilly Reich" en Core 77 (recuperado el 15 de enero de 2017) https://web.archive.org/web/20130329104451/http://www.core77.com/AWID/reich.html

${ }^{41}$ Citado en HERVAS Y HERAS, Josenia, Las mujeres de la Bauhaus: de lo bidimensional al espacio total, Buenos Aires, Nobuko, 2015.

${ }^{42}$ RÜEGG, Arthur, "Furniture and interiors: 1923 - 1925 - 1928”, en Villa La Rocca, Fondation Le Corbusier, (recuperado el 15 de enero de 2017) http://www.fondationlecorbusier.fr/CorbuCache/2049 3390.pdf

${ }^{43}$ SPARKE, Penny, Diseño y cultura: una introducción: desde 1900 hasta la actualidad, Barcelona, Gustavo Gili, 2010.

${ }^{44}$ MEADE, Martin y ELLIS, Charlotte, "Interview with Charlotte Perriand" en Architectural Review, 6 de marzo de 2014, (recuperado el 15 de enero de 2017) https://www.architectural-review.com/archive/interview-withcharlotte-perriand/8659677.article

45 DESAI, Madhavi, Women Architects and Modernism in India: Narratives and contemporary practices, London, Routledge India, 2016.

${ }^{46}$ Citado en House that was Eulie's home, Roopinder Singh, 1 de septiembre de 2009.

47 KEMPPAINEN, Jouni K., “Kotona Aalto-pöydän ääressä” en Hs Teema - Aikaa kestävä arkkitehtuuri, Helsinki, nº 1, 2014, p. 20.

48 POMBO, Fatima y RUMBIN, Paulina, “Aino Marsio-Aalto (1894-1949) the Architect of Interiors and Everyday Objects to Unveil” en Architectoni.ca 2016, Online 4, 46-59 (recuperado el 15 de enero de 2017) http://ccaasmag.org/arch-issues/arch-online4/Fatima\%20Pombo_Aino\%20Marsio-Aalto.pdf

${ }^{49}$ LOPEZ RODERO, Myriam, "Mujeres en la Sombra: Aino Marsio", en LOMBARDERO, Nuria Álvarez, Arquitectas. Redefiniendo la profesión, ArquitectAs: Primer congreso de investigación en arquitectura y género. Sevilla, 20 y 21 de marzo de 2014.

${ }^{50}$ Artek and the Aaltos: Creating a Modern World en sitio Bard Graduate Center Gallery (recuperado el 16 de enero de 2017) http://www.bgc.bard.edu/gallery/gallery-at-bgc/past-exhibitions/artek-and-aaltos.html, La muestra rescata aspectos personales y de la experiencia de ambos, de los viajes y hasta de su luna de miel. Es más humana, no se centra en los objetos únicamente. Dedica un espacio a los cuatro fundadores. 
${ }^{51}$ COLOMINA, Beatriz, "With, or without you. The ghosts of modern architecture", en BUTLER, Cornelia y SCHWARTZ, Alexandra (eds.), Modern Women: Women Artists at The Museum of Modern Art, New York, The Museum of Modern Art, 2010.

${ }^{52}$ KIRKHAM, Pat, Charles and Ray Eames: Designers of the Twentieth Century, Cambridge MA, MIT University Press, 1998.

${ }^{53}$ KIRKHAM, Pat, “Introducing Ray Eames (1912-1988)”, en Furniture History, nº 26, 1990, pp. 132- 141.

${ }^{54}$ MARICONTI, Daniele y TREMOLADA, Emilio, "Design by Franca Helg for Vittorio Bonacina”, en Vimeo, 2014, (recuperado el 15 de enero de 2017) https://vimeo.com/90060376

${ }^{55}$ Knoll International Furniture Design en el sitio VSBVenturiScottBrown, (recuperado el 15 de enero de 2017) http://venturiscottbrown.org/pdfs/KnollInternationalFurniture01.pdf

${ }^{56}$ HOWARTH, Dan, Postmodern design: Queen Anne chair by Robert Venturi and Denise Scott Brown, en Dezeen 17 de agosto de 2015 (recuperado el 15 de enero de 2017) https://www.dezeen.com/2015/08/17/postmodern-design-queen-anne-chair-robert-venturi-denise-scott-brownknoll/

${ }^{57}$ Penny Sparke en Diseño y cultura: una introducción: desde 1900 hasta la actualidad hace mención a la figura de Robert Venturi en relación al diseño de muebles omitiendo a Denise Scott Brown: "he worked on a number of Pop architectural projects and he designed a range of furniture pieces for Knoll in the early 1980s".

${ }^{58}$ HOWARTH, Dan, "There is still a lot to be learned from Postmodernism" says Denise Scott Brown en Dezeen 18 de agosto de 2015 (recuperado el 15 de enero de 2017) https://www.dezeen.com/2015/08/18/denisescott-brown-interview-still-a-lot-to-be-learned-from-postmodernism-pomo-robert-venturi/

59 "L'architecte brésilien présente l'ensemble de son oeuvre: un ensemble de trois cents photographies, plans, dessins de ses principaux projets et réalisations au Brésil et à travers le monde, une dizaine de maquettes d'architecture, des meubles créés par l'architecte, des projections permanentes de diapositives et de films". CENTRE GEORGES POMPIDOU, Nota de prensa "OSCAR NIEMEYER, Architecte", 1979 (recuperado el 15 de enero de 2017) https://www.centrepompidou.fr/media/document/be/77/be77ecccf603eaf3ad0bfb1a90d2c925/normal.pdf. El resaltado es nuestro.

${ }^{60}$ DE LAURETIS, Teresa, "La tecnología del género". en RAMOS ESCANDÓN, Carmen (comp.), El género en perspectiva. De la dominación universal a la representación múltiple, México, Universidad Autónoma Metropolitana-Iztapalapa, 1991, p. 259.

${ }^{61}$ Cini Boeri_Ghost en Youtube FiamItalia, 2013 (recuperado el 15 de enero de 2017), https://www.youtube.com/watch?v=iGENONw5jDw

${ }^{62}$ FETTERLY, Judith, The Resisting Reader: A Feminist Approach to American Fiction, Indiana, Indiana University Press, 1981.

${ }^{63}$ HOWARTH, Dan, "There is still a lot to be learned from Postmodernism" says Denise Scott Brown en Dezeen 18 de agosto de 2015 (recuperado el 15 de enero de 2017) https://www.dezeen.com/2015/08/18/denisescott-brown-interview-still-a-lot-to-be-learned-from-postmodernism-pomo-robert-venturi/

Fecha de recepción: 14 de octubre de 2016

Fecha de revisión:26 de enero de 2017

Fecha de aceptación: 8 de febrero de 2017 